\title{
COSTOS EN LA CALIDAD DE VIDA DEL PROFESOR Y SU INFLUJO EN EL RENDIMIENTO DE LOS ALUMNOS
}

\author{
Marcela Villarroel y. John Wooding \\ University of Massachussetts, USA
}

\section{INTRODUCCIÓN}

Desde el prisma cuantitativo de este estudio, pretenderemos dilucidar si aquellos Costos Personales del profesor existen, y si se asocian con el rendimiento de los alumnos. De existir esta asociación, desde un enfoque cualitativo nos preocuparemos de entender cómo sería su experiencias, y estimar si existe diferente diversidad y rango de amplitud en tales vivencias según la dependencia académica a la que pertenecen los profesores.

Nuestras hipótesis plantean que a mayores Costos del Profesor, menor es el nivel de logros de los alumnos, y a mayor Inversión del Empleador en la Calidad de Vida Laboral del Profesor, mejor el nivel de logros de los alumnos.

\section{MARCO TEÓRICO}

Los "Costos del Profesor" los abarcaremos desde el punto de vista del Desarrollo Humano Sostenible. Desde este prisma, Costos serían aquellos aspectos negativos que afectan la calidad de vida del profesor. En países en vías de desarrollo como Chile, el concepto "Calidad de Vida" tiene algunas limitantes muy bien analizadas por el Sociólogo chileno Antonio Cortés, quien plantea que el concepto se ha asentado en medio de desorientaciones que han subvalorado el sustrato cultural valórico que determina la particular mirada de las colectividades y sus individuos.

Limitados por el espacio, hemos optado por proporcionar a continuación información teórica sólo para la situación en que para una misma variable de Costos, el efecto en el rendimiento de los alumnos fue mayor y estadísticamente significativo en una dependencia administrativa sobre otra. Las variables Estatus Laboral, Salario, Labor Emocional y Alumnos/Aula, no serán mencionadas en este artículo debido a que no presentaron diferencias estadísticamente significativas entre una y otra dependencia debido a la falta de contraste entre estas; el impacto era igual o similarmente sentido por los integrantes de todas las dependencias administrativas.

Escasez de tiempo personal: Macan, Shahani, Dipboye y Phillips plantean que hay que promover conductas de manejo del tiempo. Lang y Jex y Elacqua señalan que tales conductas se asocian con bajos niveles de depresión, ansiedad y síntomas físicos. Macan aporta que estas además, se asocian con bajos niveles de tensión laboral y tensión somática como con altos niveles de satisfacción laboral.

Salud: Dos modelos destacan en esta área:- Modelo demanda-control de Karasek y Theorell, el que expresa que cuando existe alta demanda laboral por un lado y poco control personal en la tarea, por otro, la tendencia a padecer una enfermedad aumenta. El segundo es el modelo de desbalance esfuerzo- 
recompensa de Siegrest que señala un alto grado de esfuerzo -como el que requiere el trabajo docente-, precisa un alto grado de recompensa laboral y ello incide en la salud del individuo.

Trayecto: Las singulares características de la locomoción colectiva nacional (microbuses), como el alto índice de accidentabilidad, recorridos más largos que la locomoción particular, delincuencia a bordo (robos y asaltos), y falta de normativa que regule los pasajeros de pie, dejan a los usuarios más permeables al estrés, según lo señalado por Cifuentes y Molina en un estudio realizado con choferes de locomoción pública en Chile.

Recursos Físicos: Casassus lideró un estudio en una amplia muestra de 14 países latinoamericanos, entre los que se incluye Chile. Los resultados en esta área señalan que las escuelas cuentan en general con facilidades edilicias y de equipamiento relativamente aceptables y el efecto de estos materiales instruccionales en el rendimiento de los alumnos es positivo. Equipamiento básico lo tienen más del $90 \%$ de las escuelas latinoamericanas, y más del $66 \%$ cuenta con equipamientos más sofisticados.

Carga laboral: Estudios a nivel mundial descubren la asociación entre largas jornadas de trabajo con una disminuida salud psíquica (Borg), excesiva fatiga (Rosa) y síntomas de burnout (Barnett). Además, excesivas horas de trabajo se asocian a interferencias en la vida familiar en términos de conflicto de rol o fatiga, preocupación e irritabilidad (Grzywacz,). A nivel regional, el estudio de Casassus revela el influjo negativo que tiene en el rendimiento de los alumnos, el que un profesor trabaje en más de un establecimiento educacional debido a la necesidad de aumentar sus ingresos.

Violencia: Los hallazgos en esta área coinciden tanto en los estudios a nivel europeo, estaounidense y regional. "L'observatoire européen de la violencia scolaire" ha realizado importantes trabajos en esta área, entre los que destaca "Violencia nos escolas: des abordagens europeías" (Debarbieux). Este y otros estudios han puesto en el tapete una nueva forma de manifestación de la violencia escolar llamada "bullying" (o "acoso"), la cual se distingue por ser organizada, solapada y silenciosa (Froschl; Baudry). A nivel latinoamericano destaca el trabajo de Abramovay en Brasil, y los de Castro en Argentina quienes también detectan el fenómeno bullying en las escuelas de nuestra región. De sus conclusiones se desprende que el profesor actualmente se encuentra más expuesto a las consecuencias de la expresión de violencia de sus alumnos, tanto entre ellos como de estos hacia el profesor. Casassus por su parte da cuenta de que los profesores "recientemente se quejan de crecientes problemas de violencia y seguridad". De la bibliografía nacional consultada los estudios actualmente están aportando a sentar bases teóricas de acercamiento al problema y el Ministerio de Educación ha difundido a nivel nacional un documento de prevención de la violencia, aunque estrategias para su tratamiento aún no se han difundido masivamente ( Edwards, Palma, Maripangui).

Autonomía Laboral: Según los hallazgos de Karasek y Theorell, las personas responden de distinta manera a la demanda laboral según el control o autonomía laboral que posean. La investigación realizada por Wright, desde otra perspectiva, relaciona la presencia de autonomía laboral con la capacidad de ejecución, ideación y planificación que le es otorgada al sujeto.

\section{MATERIAL Y MÉTODO}

Se utilizaron complementariamente los paradigmas cuantitativo y cualitativo de la investigación. El estudio es de carácter transversal e introduce elementos descriptivos y analíticos. Los sujetos que 
participaron en este trabajo fueron 1065 profesores de escuelas chilenas pertenecientes a las dependencias pública, subvencionada y particular pagada de seis comunas de Chile distribuidas en 6 de estas localizadas en la capital y 3 en distintas provincias. La unidad de análisis a nivel cuantitativo fue cada una de las 30 escuelas incorporadas en la muestra. A nivel cualitativo, la unidad de análisis fue cada uno de los 15 profesores provenientes todos de distintas escuelas, de las 30 participantes en el estudio, distribuidos equitativamente en las tres dependencias administrativas.

Las escuelas se seleccionaron acorde al Rendimiento Académico medido a través de los puntajes de una prueba censal, nacional y estandarizadas Ilamada SIMCE (Sistema de Medición de Calidad de la Educación). Estas se ubicaban dentro de aquellas que contaban con $33 \%$ de rendimiento superior y $33 \%$ de rendimiento inferior en SIMCE, descartándose las situadas en el $10 \%$ de rendimiento superior y $10 \%$ de rendimiento inferior para disminuir la probabilidad de presencia de estigma post- test .

Fuentes de información: A nivel cualitativo, una entrevista; y a nivel cuantitativo para la variable dependiente, es el logro de resultados operacionalizado en los puntajes SIMCE obtenidos por los alumnos de Cuarto Año Básico en el año 2002 de cada escuela en: Lenguaje, Comprensión del Medio y Matemáticas. Para la variable independiente; Costos del Profesor, se utilizó la información proveída por los profesores a través de una encuesta.

Las variables de control fueron Dependencia administrativa, nivel socioeconómico y edad de los docentes.

Manejo y análisis de datos: El análisis se realizó en etapas sucesivas tendientes a encontrar evidencia que quitara sustento a las hipótesis del estudio. En todas las etapas se utilizó un nivel de significación de 0.05. En la Primera Etapa se intentó precisar la asociación entre la dependencia administrativa y los resultados de los puntajes SIMCE, utilizando modelos lineales generalizados. Para la Segunda Etapa, se intentó lograr la asociación de las variables independientes (costos e impacto de inversión) con los distintos grupos de dependencia administrativa, mediante comparación de promedios de las variables independientes de los tres grupos de dependencia, utilizando el modelo lineal generalizado. En la Tercera Etapa, se procuró la asociación entre las variables dependientes y las independientes, centradas por nivel socioeconómico y edad, mediante análisis de regresión con modelos lineales generalizados. En la Cuarta Etapa se procuró mediante las variables dependientes y las independientes centradas por nivel socioeconómico: primero, bajo el modelo lineal generalizado se utilizó el coeficiente de regresión como indicador del efecto del costo o inversión sobre puntaje SIMCE de cada test; luego, se realizó el análisis estratificado por cada grupo de dependencia administrativa. Esta última etapa se complementó con el mismo procedimiento, centrando las variables por edad.

El análisis cualitativo se hizo mediante categorización de significados y generación de significados ad hoc. Basados en la primera, se ordenó cada una de las trascripciones en 11 categorías de costos: Trayecto, Tiempo, Carga Laboral, Alumnos/Aula, Recursos Físicos, Violencia, Autonomía Laboral, Labor Emocional, Salario, Salud y Estatus Laboral. Posterior a la transcripción y análisis de las entrevistas surgieron otras 4 variables : Alimentación, Gestión, Reconocimiento a la labor docente y Perfeccionamiento, lo que totabilizó 15 variables. En esta presentación, por motivos de restricción de espacio no se presentan los hallazgos relativos a las variables Labor Emocional, Alumnos /aula, Salarios, Perfeccionamiento y Estatus Laboral. El criterio utilizado para descartar estas variables se basó en que existen otros estudios 
realizados en estas áreas en nuestra región por lo que el aporte de las otras variables se privilegió. La segunda vía utilizó contrastes, comparaciones, chilenismos, factorizaciones, y grado de plausibilidad, entre otros recursos analíticos.

Para las entrevistas, los aspectos éticos de la investigación se basaron en el consentimiento informado, garantía de confidencialidad en difusión de la identidad de la escuela, difusión de la información restringida de la extensión de la entrevista, datos demográficos identificatorios de la escuela, confidencialidad de la información para los superiores directos y acceso a la grabación y transcripción restringido sólo a la investigadora principal.

La confiabilidad se logró mediante pre test de entrevistas pilotos y chequeo de fidelidad acústica, siendo la misma persona la entrevistadora, transcriptora y analista. La confiabilidad del entrevistador se alcanzó mediante múltiples intérpretes con grado de acuerdo espontáneo del 90\%. La confiabilidad de la transcripción se aseguró por medio de la transcripción de la entrevista inmediatamente finalizada esta y utilizando la aproximación preservacionista a la información. La validez se estimó mediante triangulación (métodos cuantitativos y cualitativos). El resultado nos revela que existe concordancia en 7 de de las 11 variables analizadas por ambos métodos (63.6\%)

\section{RESULTADOS}

1. TRAYECTO: Es la variable que consigna mayor significación estadística, todos los resultados atingentes a esta variable son estadísticamente significativos para la dependencia municipal ( Lenguaje 0.0031- Matemáticas 0.0168 - Comprensión del Medio 0.0021$)^{1}$, y el influjo en los resultados negativos de rendimiento en los alumnos impacta mayormente en aquellos provenientes de tal dependencia afectándose su rendimiento en -.68 puntos en Lenguaje, -.79 puntos para Matemáticas y .-65 puntos en Comprensión del Medio cuando el trayecto es desagradable para el profesor.

Los hallazgos cualitativos coinciden en encontrar en la dependencia municipalizada los antecedentes más contundentes, pues sus profesores son quienes mayormente ocupan la locomoción colectiva para viajar, mayor tiempo utilizan en viajar, recurren a más medios mixtos para transportarse, algunos viajan en el bus de pie y cuentan mayormente con escuelas localizadas en sectores con alto índice delincuencial, lo que los hace acompañarse entre ellos para disminuir riesgos de asalto. La espera a los colegas los atrasa en su retorno al hogar aumentando su tiempo laboral. Dentro de este grupo se localizan aquellos profesores que por priorizar acceder a aranceles más económicos de la locomoción colectiva metro-, optan por viajar más temprano, desplazando a un segundo plano su desayuno. Debido a lo exhaustos que retornan al hogar, no siempre cenan y privilegian dormir. Comparados con los profesores de escuelas particulares pagadas -quienes más locomoción particular utilizan-, estos últimos extienden su tiempo familiar en traslados con miembros de la familia o se desconectan más pronto de la tarea laboral escuchando programas radiales de su preferencia al interior de sus vehículos, y su traslado lleva menos tiempo.

2. TIEMPO: En las escuelas municipalizadas, el impacto de esta variable en los resultados de los alumnos es de entre un 2.5 hasta 2.7 veces mayor respecto de las particulares pagadas y subvencionadas, destacando sólo en la dependencia municipalizada significación estadística en los resultados ( $\mathrm{L}$ 0.0247),

\footnotetext{
${ }^{1}$ En adelante, L para Lenguaje, M para Matemáticas y C para Comprensión del Medio.
} 
afectando negativamente el rendimiento de los alumnos en -1.93 puntos en Lenguaje, -1.23 puntos en Matemáticas y -1.53 puntos en Comprensión del Medio, cuando el tiempo personal y familiar del profesor es escaso.

Los hallazgos cualitativos muestran que en el nivel de escuelas municipalizadas es donde los profesores dsponen de menos tiempo personal y familiar, por lo que postergan aficiones personales y hobbies por privilegiar el tiempo familiar, duermen menos por actividades relacionadas con su profesión docente y es el único grupo donde se detecta que errores en la asignación horaria influyen tanto en la escasez de tiempo para almorzar como en perder tiempo de instrucción pedagógica por recuperar la atención de los alumnos tras bloques de noventa minutos de clases interrumpidos por recreos. Finalmente, a los profesores municipalizados es a quienes menos tiempo se les concede para preparar clases.

3. RECURSOS Físicos: Cantidad y calidad de insumos provistos para el ejercicio pedagógico presentaron valores significativos a nivel de la dependencia municipalizada donde recaen en las escuelas

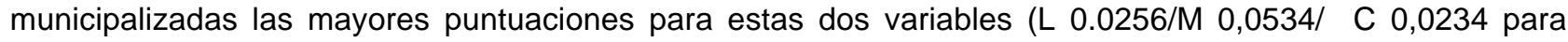
cantidad de insumos, y L0.0246/ M 0.0545/ C 0.0080 para calidad de insumos).

Llama la atención la diferencia existente en la infraestructura y provisión de insumos entre escuelas particulares pagadas. Unas cuentan con bienes inmuebles bien provistos tanto para la confortabilidad de los alumnos como del profesor, y otras cuentan con espacios mínimos para el trabajo y el confort del profesor detectándose escuelas que no cuentan con infraestructura mínima como gimnasio o colchonetas para 1200 alumnos.

La provisión de computadores por alumno varía en las escuelas particulares de 1 computador/alumno/45'/semana a 1 computador/0.225 alumno/45'/semana en las escuelas municipalizadas. La carencia de textos escolares en las escuelas municipalizadas se detecta desde la enseñanza básica, en años consecutivos sin reposición de éstos. Gran parte de los profesores entrevistados en escuelas municipalizadas señalan que recursos básicos como tinta y papel son insuficientes para cubrir las demandas de los alumnos y recursos de apoyo pedagógico como pizarras blancas no cuentan con marcadores para escribir en estas y ello debe paliarse con el aporte de los padres o la inversión particular de los profesores.

En relación al entorno físico, donde los profesores realizan su trabajo que no es de aula, es común encontrar que los profesores de escuelas particulares pagadas cuenten con edificios dotados de distintos ambientes para el trabajo y relajo docente -ambientes fumadores y no fumadores y más de una habitación adecuada para el relajo de los docentes -, pasando por escuelas subvencionadas que se han habilitado en antiguas casa-habitación y cuentan con minúsculas salas de profesores hasta llegar a escuelas que han improvisado las salas de profesores en lugares no aptos tanto en escuelas particulares subvencionadas como municipalizadas (habitación donde pernoctaba el cuidador de la escuela y una parte de un comedor de alumnos).

El contraste en el alhajamiento también es notorio. Existen escuelas particulares pagadas que cuentan con muebles, utensilios y electrodomésticos provisto para complementar la confortabilidad del profesor, en las escuelas particulares subvencionadas el mobiliario es suficiente para la demanda, y la provisión de utensilios y electrodomésticos es compartida entre el establecimiento educacional y los mismos profesores, lo mismo sucede en las escuelas municipalizadas llegándose al extremo que existen 
establecimientos en que las salas de profesores han sido amobladas y alhajadas completamente por el cuerpo de profesores, con muebles de desecho, los que ellos mismos han reparado. Sólo a nivel de escuelas municipales se detecta la prohibición de estufas durante el invierno, a no ser que el combustible sea financiado por el cuerpo docente. En todas estas aristas que tienen que ver con la provisión de recursos físicos, existe proporcionalidad directa entre las distintas variables que la componen; descubriéndose que a menores recursos económicos que tienen los profesores, mayor es el desembolso monetario que deben hacer para cubrir demandas profesionales y de mínimo confort para su desenvolvimiento docente, y viceversa (adquisición de marcadores de pizarra, papel higiénico, calefacción y jabón ).

4. VIOLENCIA: Significación estadística sólo a nivel de escuelas municipalizadas ( $\mathrm{L}$ 0.0130/ M 0.0441). Reducción de 0.98 punto en Lenguaje, 0.90 punto en Matemáticas y 0.64 punto en Comprensión del Medio. Tal evidencia es confirmada por los resultados obtenidos a nivel cualitativo. Se evidencia sólo a nivel de escuelas municipalizadas que sus profesores son los más susceptibles a ser víctimas de violencia y quienes cuentan con el menor soporte profesional provisto por sus empleadores para enfrentar tales situaciones. Entre alumnos, prevalece una forma de manifestación que responde más a impulsos, y como respuesta a una primera agresión, lo que requiere en el profesor de multiplicación sostenida del foco de atención. En las escuelas particulares pagadas es más común la manifestación llamada bullying, que se caracteriza por ser organizada, oculta, silenciosa, persistente, y poco detectable por parte del profesor.

Existen antecedentes detectados exclusivamente en las escuelas municipalizadas que explican el nivel de significación estadística existente en esta variable sólo para esta dependencia; desautorización de las decisiones disciplinarias tanto a nivel del microcosmos escolar (director o inspectores hacia profesores) como del macrocosmos escolar (empleadores hacia los directores). Inadecuadas decisiones de tipo logístico influyen negativamente en la violencia de los alumno, tales como asignar la jornada alterna a sus pares, a alumnos de enseñanza secundaria aglutinados en su curso por criterios de indisciplina; si en la mañana se desarrolla la jornada escolar secundaria, los cursos de secundaria con problemas disciplinarios deben asistir en la tarde a clases junto a la jornada de escuela elemental. Horarios de clases mal confeccionados o asignación de mobiliario de alumnos pequeños a mayores también se detectan como agentes facilitadores de manifestaciones de violencia. También se detecta que existe violencia verbal y psicológica de parte de directivos hacia profesores obstruyendo a través de la imposición jerárquica el perfeccionamiento docente como los aportes de colegas.

5. AUtONOMía LABORAL Resultados estadísticamente significativos sólo a nivel de escuelas municipalizadas (C 0.0462) y una tendencia positiva tanto en Lenguaje como Matemáticas, lo que nos estaría indicando que cuanto a mayor autonomía laboral se otorgue a los profesores, el rendimiento académico de los alumnos mejora.

Mientras en las escuelas particulares pagadas, los profesores perciben que existe confianza en su capacidad pedagógica y se estimula la autonomía, en las subvencionadas la autonomía laboral se gana si el profesor demuestra un buen desenvolvimiento laboral y en la municipal no hay atribuciones positivas a la autonomía laboral, sino es consecuencia azarosa del abandono y desidia que los profesores perciben de parte de las autoridades educativas frente a su tarea. Paralelo a ello existe un bajo nivel de control personal en las decisiones de ejecución relativas a la disciplina de los alumnos, evaluación y labores administrativas; plano en que son fuertemente controlados por los directivos, y restringida en este aspecto la habilidad que 
los profesores sienten poseer para resolver este tipo de conflictos. Se agrega a lo anterior que sólo a nivel municipal los directores son desautorizados en sus decisiones administrativas por sus empleadores.

6. CARGA LABORAL: Aunque los valores no son estadísticamente significativos, los más cercanos se presentan en la administración municipal.

Los detonantes de sobrecarga laboral se registran en la dispersión de establecimientos laborales en que se trabaja, siendo los profesores de escuelas pagadas quienes laboran en menos establecimientos y los de escuelas municipalizadas en más. Los profesores sienten que adaptarse de un establecimiento educacional a otro demanda mayor trabajo emocional. En las escuelas particulares pagadas la dispersión horaria es mayor abarcando más niveles, sólo en una asignatura, la de especialidad del profesor. A nivel municipalizado es menor la dispersión de niveles, pero mayor la miscelánea de asignaturas focalizadas en un profesor, a veces no coinciden con su nivel de especialización, otras se imponen asignaturas sin considerar experiencia, y los directivos intentan paliar tal problema delegando la tarea de apoyo en los colegas especialistas durante su tiempo libre. En educación parvularia y primeros años básicos no siempre se cuenta con personal auxiliar en aula, y hay demanda adicional de trabajo con la incorporación de nuevos programas como el de Integración Escolar de alumnos con discapacidad, donde la carencia de recursos diagnósticos, de apoyo familiar, ortopédicos y pedagógicos para tales alumnos es suplida con esfuerzo adicional demandado al profesor.

La carga no lectiva en las escuelas particulares se centra en la preparación de clases y labores administrativas consideradas excesivas. En las particulares subvencionadas se suma a ello el realizar turnos cuidando alumnos en recreos, y reemplazar a colegas durante sus ausencias realizando tareas de vigilancia de alumnos en aula. En las escuelas municipalizadas se suma a todo lo anterior el realizar talleres y cursos tras la jornada escolar (Club de Teatro, ajedrez, deportivos, etc.) y coordinar programas (prevención de drogas y alcoholismo).

7. ALIMENTACIÓN: A nivel de escuelas particulares pagadas y subvencionadas los profesores cuentan mayoritariamente con comidas provistas por sus establecimientos educacionales, en las municipalizadas en la mayor parte de los casos se la proveen los propios profesores aunque cuentan con un item en su salario que comprende alimentación. Coincide que a nivel de establecimientos particulares subvencionados y municipalizados los profesores que obvian una de las comidas sean quienes trabajan en más de una escuela, lo que se traduce en que quienes más demanda laboral tienen sean los peor alimentados durante la jornada laboral y sus efectos en el rendimiento de los alumnos se expanden a una mayor población estudiantil debido a que los cursos más numerosos provienen de tales dependencias administrativas.

8. GESTIÓN: Los datos cualitativos revelan una asociación entre idoneidad del personal directivo con facilitación u obstaculización del quehacer de los profesores. A mayor idoneidad, mayor facilitación a través del cargo de la tarea docente, y a menor idoneidad, mayor su obstaculización.

Conductas detectadas en directivos como desinterés en el bienestar docente, mayor cantidad de trabas para el ejercicio pedagógico, trabas a la formación docente, y absurdos administrativos provienen de directivos de escuelas municipalizadas que son precisamente quienes cuentan con el menor grado de formación e idoneidad profesional para ejercer su cargo. 
9. RECONOCIMIENTO: A nivel municipal se detectó una escuela acreedora de la asignación de excelencia pedagógica por tercer año consecutivo, observándose un positivo impacto en la comunidad docente por tal distinción. Pudimos constatar que la experiencia de los profesores con distinciones cuando surgen de iniciativa propia y no por nominaciones de colegas, es que para postular a estas se debe saltar muchas vallas de índole burocrático que finalmente hacen que desistan de postular por no contar con el tiempo suficiente para cumplir con todos los requisitos requeridos.

Donde se presenta el mayor abismo entre esfuerzo desplegado y recompensa otorgada es en la dependencia municipalizada, detectándose no sólo ausencia de recompensa positiva al esfuerzo desplegado y buenas iniciativas, sino, además, en ciertos casos se otorga recompensa negativa, o positiva a quienes cuentan con simpatía de dirección por asuntos no ligados al quehacer pedagógico.

10. Perfeccionamiento: En todas las dependencias existen algunos sostenedores que imponen áreas de desarrollo, coartando la capacidad de elección de los profesores. En general, para el financiamiento en las tres dependencias administrativas se utiliza la modalidad de reembolso que consiste en que el profesor debe pagar su capacitación con sus propios recursos y una vez terminado y aprobado, le es devuelto su valor. Esto, a juicio de algunos profesores municipalizados, es un impedimento para perfeccionarse, pues no cuentan con dinero para hacerlo.

Si bien existe la gratificación monetaria por el Perfeccionamiento, se entrega actualmente más a los profesores provenientes de escuelas municipalizadas que a los de las otras dependencias administrativas, tales pagos se ejecutan con atrasos de hasta dos años.

Las limitantes económicas y de tiempo para acceder a los cursos de perfeccionamiento son barreras mencionadas sólo a nivel de escuelas municipalizadas, por lo que la población más joven es quien más participa de estos. En tales escuelas se detecta que los profesores declaran la inutilidad de algunos cursos de perfeccionamiento que no responden a sus demandas y hay otros cursos como los que se ofrecen en el área de Computación que sí son demandados por ellos, pero no proporcionan las habilidades que necesitan, y finalmente terminan sin adquirir el conocimiento y pericia requeridos. Los profesores no estarían usando software e Internet para preparar clases tanto por la deficiente capacitación que afirman tener como por el difícil acceso que tienen a computadores destinados a su uso exclusivo, y el escaso tiempo con que cuentan para preparar sus clases. Esto también incide en que la incorporación de Tecnologías se resiente mayormente a nivel municipal, y por ende la adquisición de la cultura informática tanto en alumnos como profesores.

11. SALUD: Valores estadísticamente significativos para este item se encuentran sólo a nivel de la dependencia municipal ( $L$ 0.00213/ C: 0.0358). El impacto en el rendimiento académico de los alumnos es mayor en quienes provienen de esta dependencia.

En todas las dependencias administrativas surgen atribuciones y síntomas comunes, aunque las afecciones están presente en un porcentaje mayor en los profesores provenientes de escuelas municipalizadas. Entre las primeras se menciona la violencia de alumnos y apoderados y la escasez de herramientas que tienen para enfrentarla. Entre los segundos los más recurrentes son disfonías, lumbago crónico, tabaquismo, colon irritable e insomnio.

Las atribuciones de las dolencias físicas, ruido por traslado de mobiliario, deficiente alimentación, escaso tiempo para ello, inadecuada infraestructura e imperfección del mobiliario, son las más 
recurrentemente mencionadas. Afecciones físicas derivadas de aquello son deficiencias auditivas, obesidad, neumonías, lumbago. Ambas mayormente mencionadas por profesores de escuelas municipalizadas.

\section{DISCUSIÓN}

Volviendo a lo planteado en el inicio de nuestro estudio, tras el análisis y discusión de nuestro resultados, corroboramos lo sostenido por Antonio Cortés, en este caso en lo concerniente al Desarrollo Humano Sostenible en Educación; la carencia de una calidad de vida laboral del profesor que respete su entorno idiosincrásico, su sustrato cultural y valórico. Descubrimientos hechos en este trabajo, reveladores de algunos aspectos de la calidad de vida consustanciales a nuestra idiosincrasia en sus peculiares formas de manifestación y de impacto, tales como trayecto, alimentación, carga laboral, recursos físicos, violencia, autonomía laboral, perfeccionamiento y reconocimientos a la labor docente deben ser considerados en su conceptualización y operacionalización.

Existe un alto grado de resultados estadísticamente significativos en las variables a nivel de la dependencia administrativa municipalizada, aún luego de controlar por edad y nivel socioeconómico, estratificado por nivel de dependencia. Ello nos lleva a sostener que los resultados son sólidos aún cuando el tamaño de la muestra es pequeño (30 escuelas).

El trayecto desagradable hacia y desde la escuela, la escasez de tiempo personal y la percepción de salud deteriorada a nivel mental y corporal impactan más fuertemente en el rendimiento de los alumnos de las escuelas municipalizadas. Esta apreciación del Trayecto avala en parte lo planteado por Cifuentes y Molina, quienes describen que la peculiaridad de la movilización colectiva chilena incide en a mayor permeabilidad al estrés de sus usuarios.

La mayor escasez de tiempo personal no se vería resuelta en este caso por las propuestas de conductas de manejo de tiempo de Macan, Shahani, Dipboye y Phillips. Estas existen, pero no logran los efectos reducidores de tensión que describen Lang y Jex y Elacqua, pues para lograrlos, tales conductas deben acompañarse de una dotación de tiempo suficiente para ejecutar las tareas de la que los profesores municipalizados no disponen.

La explicación para la deteriorada salud de los profesores de escuelas municipalizadas coincide con lo mencionado en el modelo demanda control de Karasek y Theorell, al evidenciar que son ellos quienes tienen mayor demanda laboral con un menor control personal en esta, desencadenando este cruce mayor permeabilidad al deterioro en la salud. Esto se refuerza con el otro modelo explicativo de la salud docente; desbalance esfuerzo-recompensa de Siegrest, donde el menor grado de recompensa al esfuerzo de la tarea docente se encuentra en los profesores municipalizados.

Desde el punto de vista de Wright, la baja autonomía laboral del profesor municipalizado evidenciada en su restringida capacidad de ejecución- situaría su quehacer en el último eslabón de la estructura de clase, lo que afecta directamente su estatus laboral.

La cantidad de insumos que se proveen, como la calidad de estos, coincide con los hallazgos de Casassus a nivel de las escuelas públicas latinoamericanas en el sentido de que es más pequeña en las escuelas públicas, llamadas en Chile municipalizadas, sin embargo no son coincidentes en lo relativo a que el equipamiento básico (como textos, papel, tinta y marcadores de pizarra blanca) existen más del $90 \%$ de las escuelas públicas latinoamericanas, y en las nuestras este porcentaje es marcadamente inferior. Acorde 
a los resultados de la investigación de Casassus en lo relativo a este item, el aumento en recursos incide en el rendimiento en Lenguaje. Extrapolando a nuestros resultados, la mayor privación de materiales en la dependencia municipalizada produciría mayor descenso en el rendimiento de lenguaje de estos alumnos, lo que coincide con nuestros hallazgos.

Los profesores más lábiles, a los efectos de la violencia al interior de sus establecimientos educacionales son los provenientes de escuelas municipalizadas, siendo sus alumnos los mayormente afectados en su rendimiento por esta variable. Nuestros hallazgos coinciden con los de Debarbieux y Blaya, Abramovay, Froschl, y Sprung y Castro en lo concerniente a que también detectamos presencia del fenómeno bullying y que el profesor actualmente está más expuesto a manifestaciones de violencia entre alumnos o de ellos hacia el profesor. Nuestros hallazgos nos indican que en la depedendencia municipalizada es notorio cómo el fenómeno de la violencia se expande más allá del marco profesor alumno-apoderado, extendiéndose a los empleadores y directores hacia los profesores con manifestaciones de violencia de tipo psicológica y verbal.

La carga laboral al ser mayor en intensidad en los profesores de las escuelas municipalizadas explicaría el mayor grado de disminución de salud psíquica del profesor de escuela municipalizada, consolidado esto en los hallazgos de Borg y Kristensen y Rosa.

La investigación en el área de Nutrición proveniente de países más desarrollados que Chile y que tienen un elevado nivel de investigación, intenta resolver sus propias inquietudes dentro de las cuales no está considerar el vínculo entre alimentación y rendimiento laboral, por estar este medianamente resuelto, lo que revela esta es una faceta que requiere ser explorada en nuestros países. Publicaciones recientes a nivel europeo, como las de Pryer y Martikainen revelan que se han descubierto patrones de dieta que relacionan una baja ingesta de nutrientes y alta ingesta de carbohidratos con el nivel sociocultural de las personas, asociándose éste a empleos no profesionales con escaso nivel de escolarización lo que no les permite distinguir entre comidas saludables de las que no lo son.

A diferencia de los estudios precedentes, nuestros hallazgos nos señalan que los profesores de escuelas municipalizadas coinciden con ser quienes consumen una dieta de tales características debido al escaso tiempo provisto para almorzar, escaso salario y errores en la asignación horaria, y no por ignorancia de los beneficios de una dieta saludable como lo revelan los anteriores estudios realizados en países con características culturales y económicas distintas al nuestro.

Es destacable cómo en lo que respecta a la variable Perfeccionamiento las demandas de actualización en el área de Computación no han sido satisfechas en lo que respecta a los profesores de escuelas municipalizadas, generándose cursos que no logran alfabetizar en esta área a los profesores de antiguas generaciones docentes. A ello se suma que el uso de software e Internet no llega a ser tal por la escasez de medios existentes ( 9 computadores para todo el alumnado en escuelas municipalizadas con matrícula superior a 600 alumnos). Tales antecedentes evidencian que los objetivos del Programa Enlaces, encargado de la difusión a nivel escolar y docente de las tecnologías de la información, en cuanto a gestión y cobertura no estarían cumpliéndose con la cabalidad e intensidad requerida para ello. Se suma a lo anterior que nuestros hallazgos nos han mostrado que sólo a nivel de escuelas municipalizadas los profesores acusan obstáculos económicos y de escasez de tiempo para no perfeccionarse, y también se detectan obstáculos de parte de la dirección de las escuelas para que los profesores accedan al 
Perfeccionamiento Académico. Todo ello redunda en que los alumnos que menos acceso tienen a herramientas pedagógicas renovadas sean los procedentes de escuelas municipalizadas.

Se logra detectar que tiene efectos positivos en el profesor municipalizado el Recononocimiento público a su labor docente mediante la asignación de excelencia pedagógica otorgada por el Ministerio de Educación, sin embargo es en esta dependencia donde se detecta el mayor abismo entre el esfuerzo desplegado por el profesor y la recompensa otorgada, lo que según Siegrest les haría más vulnerables a padecer afecciones de salud. Ello se corrobora en nuestro trabajo, donde los profesores de esta dependencia administrativa son los más afectados en su salud psíquica y corporal.

La bibliografía consultada referida a Gestión Educativa coincide en afirmar que el rol del Director es preponderante en la interacción escolar positiva. Casassus señala que aquellos alumnos que asisten a escuelas donde sus directores tienen un liderazgo positivo, tienen un mejor rendimiento que aquellos que no. García Huidobro también confiere al Director un rol clave en asegurar la eficacia pedagógica de su institución escolar a través de un modelo de interacción democrática donde los profesores puedan tomar decisiones respecto a la organización de la enseñanza.

Los resultados arrojados por nuestro estudio asocian entre idoneidad del personal directivo y facilitación u obstaculización del quehacer de los profesores. Así, a mayor idoneidad del Director y a nivel macroinstitucional, del empleador, mayor facilitación a través del cargo de la tarea docente, y a menor idoneidad; mayor obstaculización de esta. Todo ello coincide con la información proporcionada por la bibliografía consultada. Sin embargo, un nuevo hallazgo surge de nuestro estudio al constatar que la distribución de los Directores menos idóneos está lejos de ser equitativa y se concentra sustantivamente en las escuelas municipalizadas, las cuales son las más afectadas tanto en el rendimiento de los alumnos como en el desempeño de sus profesores.

Ante la evidencia de la información discutida aquí, podemos afirmar que los Costos del Profesor existen y se manifiestan con distinta magnitud según la dependencia a la que pertenezcan los profesores, resultando más afectados los alumnos de más escasos recursos, quienes constituyen la mayor población escolar chilena.

\section{CONCLUSIÓN}

Los múltiples análisis realizados no nos proporcionaron evidencias que permitieran descartar nuestras hipótesis; a mayores Costos del profesor, menor el nivel de logro de los alumnos. Y, a mayores inversiones del empleador, mejor el nivel de logros de los alumnos.

\section{BIBLIOGRAFÍA}

ABRAMOYAV, M. Escola e violencia, Brasilia, UNESCO, 2002.

BAUDRY, P. Souffrances et violences a l'adolescence. Qu'en penser ? Que faire ?, Paris. Ministry delegue a la Ville, 2000.

BORG, V., KRISTENSEN, T. Psychosocial work environment and mental health among travelling sales people. Work \& Stress, (13), 132-143,1999.

CASASSUS, J. La escuela y la (des)igualdad. Santiago, Lom, 2003. 
CAStRO, A.. Cuando prevenir la violencia no basta. Revista lberoamericana de Educación (versión electrónica) http://www.campus-oei.org/revista/,2004.

CAStRo, A. Desaprender la violencia. Un nuevo desafío educativo. Buenos Aires, Bonum,2004.

Cifuentes, M., MolinA, G. Trabajo y salud mental del conductor de la locomoción colectiva. Ponencia presentada en el Tercer Congreso Chileno de Epidemiología. Valparaíso, Chile, 1998.

CORTES, A. Calidad de Vida en Chile: Reflexiones críticas sobre las mediciones, Revista de Salud Pública, 44(4),2002.

Debarbieux, E., Blaya, C. Violencia a l'ecole et politiques publiques.Paris, Unesco, 2001.

Edwards, L., Palma, R., Maripangul, C. Política de convivencia escolar. Santiago. http//www. mineduc.cl,2003

FROSHL, M., SPRUNG, B. Quit it! A teacher's guide on Teasing and Bullying for use with students in grades K3. New York, Spiral,1998.

GARCIA-HUIDOBRO, J . La centralidad de la escuela en la política educativa chilena de los años noventa. En Políticas educacionales en el cambio de siglo. La reforma del sistema escolar de Chile. Cristian Cox (Ed.), 253-315,2003.

GoDIN, I., KITTEL, F. Differential economic stability and psychosocial stress at work: associations with psychosomatic complaints and absentism. Social Science \& Medicine, 58, 1543-1553, 2004.

GRZYWACZ, J., MARKS, N. Reconceptualizing the work-family interface: An ecological perspective on the correlates of positive and negative spillover between work and family. Journal of Occupational Health Psychology, 5(1), 111-126, 2000.

JEX, S., ELACQUA T. Time management as a moderator of relations between stressors and employee strain. Work\&Stress, 13(2) 182-191, 1999.

KARASEK, R., THEORELL, T. Healthy work: Stress, Productivity and the Reconstruction of Working Life. New York: Basic Books, 1990.

LANG, D. Preventing short-term strain through time management coping. Work\&Stress, 6, 169-176,1992

MACAN, T. Time management: test of a process model. Journal of Applied Psychology, 79, 381-39,1994

MACAN, T., ShahANI, C., DIPBOYE, R. y PHILLIPS, A. College student's time management: Correlations with academic performance and stress. Journal of Educational Psychology, 82, 760-768, 1990

MARTIKAINEN, P. Socioeconomic differences in dietary patterns among middle-aged men and women. Social Science \& Medicine, 56, 2003, 1397-1410, 2003

Merllie, D., PaOl, P. Dix ans de conditions de travail dans L'Union européenne. Doblin: Doblin Fondation Européen pour l'amélioration des conditions de vie et de travail, 2000

PRYER, L. Dietary patterns among a national random sample of British adults, Journal of Epidemiology and Community Health, 55, 29-37, 2001

ROSA, R. Extended workshifts and excessive fatigue. Journal of Sleep Research, 4(2), 51-56, 1995 
SIEGREST, J. Adverse Health effects of high effort-low reward conditions at work. Journal of Occupational Health Psychologyl, 1, 27-43, 1996

Wright, E., Hachen, D., Costello, C y Sprague, J. The American class structure. American Sociological Review. 47, 709-726,1982 


\title{
Contactar
}

Revista lberoamericana de Educación

\author{
Principal OEI
}

